\title{
Feeding Challenges in Patients with Esophageal and Gastroesophageal Cancers
}

\author{
Daniel Reim Helmut Friess \\ Department of Surgery, Klinikum rechts der Isar, Technische Universität München, Munich, \\ Germany
}

\author{
Key Words \\ Esophageal cancer · Feeding challenges - Gastroesophageal cancer
}

\begin{abstract}
Background: Patients undergoing treatment for esophagogastric or esophageal cancer are exposed to a considerably high risk of malnutrition due to early obstruction of the gastrointestinal passage. Presently most of the patients undergo modern multimodal therapies which require chemoradiation or chemotherapy ahead of surgery. Therefore reconstruction of the obstructed gastrointestinal passage is considerably delayed. Surgery as the only curative option after neoadjuvant treatment is the mainstay of therapy in this setting. However, many patients are at risk for the development of postoperative complications associated with the complexity of the surgical procedure. Therefore enteral feeding as a prerequisite to avoid malnutrition represents a special therapeutic challenge. Summary: This review describes the recent literature on the incidence and influence of perioperative malnutrition on oncologic outcome, measures to determine patients at risk, possible strategies to reduce or avoid malnutrition by supportive enteral/parenteral nutrition, implementation of the enhanced recovery after surgery programs and feeding routes, but also surgical and adjuvant procedures in the curative and palliative setting for patients undergoing treatment for gastroesophageal cancers. Key Messages: Appropriate identification of patients at risk is crucial to avoid malnutrition. Early nutritional interventions during multimodal/neoadjuvant treatment may be beneficial for weight loss reduction although the evidence is not conclusive. Pouch reconstructions during surgery should be applied in order to increase quality of life and eating capacity. Reduction of postoperative complications could provide potential benefits. In palliative patients, insertion of self-expanding metal stents can reduce dysphagia and improve quality of life, but does not prolong overall survival. Further evidence is required to determine the value of the procedures and measures described in this review. Practical Implications:
\end{abstract}


Nutritional risk scoring should be performed for every gastroesophageal cancer patient. Sophisticated reconstruction methods and early recovery programs should be enforced to reduce perioperative starvation periods. Self-expanding metal stents should be used for palliative patients.

(C) 2016 S. Karger AG, Basel

\section{The Medical Problem}

Nutritional problems, malnutrition and loss of appetite represent important issues in any anti-cancer treatment and may lead to metabolic and physiological changes that can negatively influence treatment outcomes. Not only the anti-cancer treatments themselves but also the cancer disease are considered to be consuming. Due to the lack of screening programs many cancers are diagnosed in advanced stages. Many patients seek medical advice only after unintended extensive weight loss, which may have already led to severe impairments in nutrient supply, vitamins and trace elements [1]. The consequences are impairments of important functions of the musculoskeletal, gastrointestinal, neuronal and immune systems, which themselves can lead to progressive deterioration and progression of the malignant disease. Malignancies in the esophagus and gastroesophageal junction represent an even greater therapeutic challenge in this setting because the gastrointestinal passage may be obstructed early in the course of the disease, leading to early malnutrition [2]. Esophageal malignancies are described to be the sixth leading cause of cancer-related mortality and the eighth most common cancer worldwide [3]. Around 450,000 patients are affected by the disease. Whereas squamous cell cancer is more dominant in Eastern Asian countries, the Middle East as well as Southern and Eastern Africa, adenocarcinoma can be considered the predominant type in Western countries, with a markedly increasing incidence [4]. Squamous cell cancer is often related to low socioeconomic status, alcohol and tobacco consumption, in contrast to adenocarcinoma, which is related to obesity, reflux and most importantly Barrett's metaplasia. Esophageal cancer is mostly diagnosed in advanced stages, when patients already present with dysphagia and unintended weight loss. Early stages usually are diagnosed incidentally. Therefore most of the patients receive multimodality therapies in order to downstage the tumor ahead of surgical resection. These modalities include chemotherapeutic regimens next to chemoradiation therapies and have been extensively evaluated in clinical trials [5-14]. These patients represent an extraordinary therapeutic challenge, because they are not only malnourished due to the advanced disease, but during preoperative therapy, nutritional status often deteriorates because the esophageal passage continues to be occluded and perioperative therapy even worsens the patient's general constitution due to chemotherapeutic side effects on the gastrointestinal tract and the immune system. Not only chemotherapy-associated nausea and vomiting, diarrhea, dryness of mouth, different perception of taste and smell, but also psychosomatic factors related to disease coping may all contribute to a reduced intake of nutrients [15]. Further, increased chemotherapy-associated toxicity was described in malnourished patients due to reduced concentrations of plasma proteins [16]. Non-metastatic patients usually undergo surgical resection either after multimodality therapy in advanced stages or directly after diagnosis in early stages [4]. The surgical procedure is chosen depending on the tumor location and the clinical stage and is either an abdominothoracic approach (Ivor-Lewis procedure) in case of squamous cell cancer of the thoracic esophagus and type I and II adenocarcinomas of the gastroesophageal junction (AEGI/AEGII), or a transabdominal approach for AEG type III. Surgical modifications may be applied in early stages, such as the Merendino procedure [17] by which the distal esophagus and the gastric fundus are resected along with 
the peritumoral lymph nodes. Here the gastrointestinal passage is reconstructed by interposing a jejunal segment. Nonetheless, the reconstruction of the gastrointestinal passage by either a gastric pull-up after esophagectomy or a reconstruction with jejunum after gastrectomy leads to impaired gastrointestinal functions, which in turn may lead to enhanced malnutrition due to reduced nutrient intake, especially in the postoperative period. This problem may even be enhanced in case of postoperative complications, requiring fasting periods. Thirty-day morbidity rates after these procedures were reported to be up to almost $50 \%$ [18], with leakage rates of 3-30\% in modern series [19]. Long-term complications like anastomotic strictures and bile reflux can lead to suspended reduction of food consumption. The treatment of palliative or unresectable patients may be even more challenging because obstruction of the esophageal passage is irreversible in most cases and therefore special aspects of nutrition have to be taken into account.

This review therefore addresses possibilities to improve nutritional status in the challenging therapeutic setting of esophageal and gastroesophageal cancer patients.

\section{Incidence and Severity of Weight Loss in Patients with Esophagogastric Malignancy}

Weight loss is a common symptom in esophageal cancer patients. The median weight loss is the highest reported in esophageal cancer patients [20] compared to other oncologic entities. A large American series reported that $57 \%$ of patients presented with weight loss at the time of diagnosis [21]. A Swedish cohort study found that weight loss persisted at least 3 years after surgery. The mean weight reduction was reported to be $10.8 \mathrm{~kg}$ [22]. There was an increased risk of weight loss in female patients and those receiving neoadjuvant chemotherapy. Tumor stage, location, histology, anastomotic type and reconstruction route were not related to the risk of postoperative malnutrition [23]. In summary, weight loss pre- and postoperatively is a relevant clinical and often underestimated problem for patients undergoing treatment for (gastro-)esophageal cancer.

\section{Influence of Weight Loss on Oncologic Outcome}

Malnourished patients undergoing gastrointestinal surgery are considered to be at risk for higher morbidity and mortality [24]. The risk of malnutrition among elective gastrointestinal surgery patients has been accounted for up to $14 \%$. Among those, $40 \%$ have been reported to suffer from postoperative morbidity [25].

Although there is a general opinion in the literature regarding negative outcomes related to pretherapeutic weight loss in cancer patients, the existing evidence does not support a connection between preoperative weight loss and oncologic outcome in general. A metaanalysis on 1,414 patients suffering from various kinds of cancers having been randomized in thirteen different studies found that nutritional support in malnourished patients improved nutrient intake and some quality of life aspects, but not cancer survival [26]. However, all these studies demonstrated marked heterogeneity, which is why that meta-analysis does not allow for definite conclusions on esophageal and gastroesophageal cancer patients.

The influence of preoperative weight loss on postoperative complications and survival was investigated in several studies for patients undergoing esophagectomy for cancer. Van der Schaaf et al. [27] demonstrated that $17 \%$ of patients had severe weight loss preoperatively. Severe weight loss was defined as a reduction of $>10 \%$ of the regular weight before any treatment. These patients had a decreased 5-year survival after surgery (HR 1.34, 95\% CI 
1.02-1.74) without increased risk of postoperative complications. A meta-analysis from China investigating the impact of BMI on postoperative and oncologic outcomes in 2,031 patients found that higher BMI was associated with higher frequency of postoperative complications but improved overall survival [28]. Another study by Skipworth et al. [29] produced contrary results and found that preoperative weight loss was not associated with poor prognosis and that postoperative complications were not increased. On the other hand a Japanese retrospective analysis revealed weight loss of $>2 \%$ to be associated with negative influence on postoperative survival (HR 1.64, 95\% CI 1.09-2.46, $\mathrm{p}=0.001$ ) [30].

A recent analysis by Filip et al. [31] demonstrated that nutritional status can be a prognostic factor for postoperative complications. However, the authors used the prognostic nutritional index, which is not commonly applied in nutrition assessment. Serum albumin was proposed as a surrogate marker indicating malnutrition. A review by Goh et al. [32] concluded that this marker may not be suitable for prediction of postoperative complications due to the heterogeneity of the studies and that nutritional interventions based on serum albumin level should be considered with care.

Conclusively, a clear correlation from an evidence-based standpoint cannot be determined by the data presented. Nonetheless most of the analyses report a correlation between preoperative weight loss, postoperative morbidity and overall survival.

\section{Nutritional Status of Gastroesophageal Cancer Patients and Malnutrition Assessment}

Determination of patients at risk for malnutrition is considered to be of utmost importance to identify the risk of weight loss in patients with esophageal and gastroesophageal cancers. A systematic review by Skipper et al. [33] reported on the evaluation of eleven different previously published assessment tools for patients who were treated in an acute hospital care setting and concluded that the Malnutrition Screening Tool was the only tool to be valid and reliable for malnutrition assessment in comparison with the Subjective Global Assessment (SGA), which is considered to be the gold standard [33]. The Nutritional Risk Score (NRS) provided stable data, but did not reveal statistical robustness. However, these validation studies were unspecific and applied to a general acute hospital care situation; they were not tailored to cancer patients, especially not to esophageal and gastroesophageal cancer. No evaluation or analysis was performed on the Malnutrition Screening Tool or the NRS for esophageal cancers. However, there are limited data on the NRS for gastric cancer patients [34]. Ryu and Kim [35] demonstrated a correlation between the SGA and the NRS 2002. However, most of the patients in this analysis received subtotal gastrectomies, which does not reflect the clinical reality in esophageal surgery. A retrospective study by Gavazzi et al. [36] demonstrated that the NRS may be correlated to stage and quality of life in gastric cancer patients. Due to the lack of data there is no 'gold' standard for the assessment of nutritional status in esophageal and gastroesophageal cancer patients from an evidence-based point of view. A Cochrane meta-analysis found that due to the heterogeneity of studies and lack of randomized controlled trials, there is no evidence of the effectiveness of nutritional screening [37]. Another systematic review by van Bokhorstde van der Schueren et al. [38] found that not a single nutrition assessment tool was capable of adequate screening and prediction of hospital outcome. Nonetheless the authors found that the SGA, the NRS 2002 and the Malnutrition Universal Screening Tool performed adequately in outcome prediction for adults but not for elder patients. The European Society for Clinical Nutrition and Metabolism (ESPEN) recommends the NRS 2002 score for surgical patients as a standard tool [39]. 


\section{Enteral versus Parenteral Nutrition}

Considering the data discussed so far, early recovery from perioperative weight loss appears to be beneficial for malnourished patients or patients at risk in order to reduce perioperative morbidity and oncologic outcome. Two major concepts are described in the literature: enteral and parenteral nutritional support. However, meta-analyses and systematic reviews on the outcome of those interventions are inconclusive so far. A meta-analysis by Peter et al. [40] investigating 30 randomized controlled trials demonstrated that there was no difference in mortality between the parenteral and enteral groups, whereas parenteral nutrition was associated with increased infectious complications, catheter-related infections and longer hospital stay. However, the authors concluded that due to the heterogeneity of the enrolled trials, a clear statement cannot be made and further investigation is necessary [40]. A recently published randomized controlled trial investigating differences between enteral and parenteral routes in intensive care patients concluded that there was no difference between the two procedures regarding infectious complications [41]. Another randomized controlled trial investigated the application of parenteral nutrition in intensive care patients unable to receive early enteral nutrition and demonstrated no difference in 60-day mortality rates compared to those patients undergoing standard care with the benefit of shorter periods of invasive ventilation and less muscle and fat loss [42]. This setting does however not reflect the clinical reality in esophageal and gastroesophageal cancer surgery. A recently published meta-analysis of ten prospective studies investigated the outcomes of parenteral or enteral nutrition after esophagectomy [43]. There was no significant difference in overall postoperative complication rates, but pulmonary complications and anastomotic leakages $(-54 \%)$ were significantly reduced in the enteral nutrition group. Postoperative albumin levels did not differ significantly between the groups but increased faster in the enteral nutrition group. The results should be considered with care in the Western hemisphere because the metaanalysis included only randomized trials from China and Japan.

\section{Enhanced Recovery after Surgery}

Despite the fact that the evidence levels for nutritional interventions do not necessarily support the notion that early feeding reduces postoperative complications and in consequence may be helpful to control body weight in esophageal and gastroesophageal cancer patients, improvement of postoperative care should be considered a prerequisite in order avoid malnourishment. Despite this, postoperative complications have been associated with decreased oncologic overall survival after cancer surgery [44-46]. Several complications may arise in gastroesophageal cancer surgery, among them anastomotic leakages and pulmonary complications requiring intensive care treatment in many cases. So-called enhanced recovery after surgery (ERAS) programs were proposed in the past [47]. Despite marked heterogeneity between the programs, all found reduced morbidity and mortality. A recently published review summarized the results and reviewed the respective components of the ERAS programs.

\section{Preoperative Period}

Reduction of fasting times to $6 \mathrm{~h}$ for solid food and to $2 \mathrm{~h}$ for liquids is recommended. Further, carbohydrate loading could attenuate surgical stress. Preoperative improvement of nutritional status was not recommended due to lack of data and unavailability of randomized trials. The same applies for preoperative inspiratory muscle training in esophageal surgery. Despite lack of high-quality evidence-based data, improvement of preoperative iron deficiency anemia by iron supplementation is supported by existing data. 


\section{Perioperative Period}

Epidural pain control was found to be beneficial for postoperative outcomes. The application of minimally invasive techniques is recommended but not based on high-quality data. Goal-directed therapy of perioperative fluid status is recommended for reduction of pulmonary complications.

\section{Postoperative Period}

Removing the chest drain was recommended at a daily discharge $<200 \mathrm{ml}$ without a significant risk of fluid collections. Further, limitation to only one drain was recommended. Interestingly gastric conduit decompression by a nasogastric tube was recommended. Further, early removal of urinary catheters was recommended to reduce local infectious complications. Besides this all patients should be mobilized early in the postoperative course.

\section{Feeding Routes}

Enteral feeding in post-esophagectomy patients represents a major challenge because surgeons are usually reluctant to apply early oral food intake due to a possible risk for anastomotic breakdown. Therefore, several enteral feeding routes were investigated in clinical trials. Most commonly nasoduodenal tubes were compared to jejunostomy catheters in the past. A randomized controlled trial by Torres Junior et al. [48] randomized 42 patients to receive either nasoenteric tubes or jejunostomy catheters after esophagectomy. Although there was no difference in initiation of enteric nutrition, the time of enteral feeding was significantly shorter in the nasoenteric tube group and parenteral nutrition had to be applied more frequently. The route-dependent complication rates did not differ significantly. Therefore the authors concluded that a feeding jejunostomy was favorable in the light of possible postoperative complications because enteral feeding did not have to be suspended in case of anastomotic complications. Another retrospective analysis on 262 patients concluded that needle catheter jejunostomy insertion was a safe procedure with a low complication rate $(1.5 \%)$ enabling long-term enteral feeding from the first postoperative day [49]. Han-Geurts et al. [50] also investigated the application of feeding jejunostomies compared to nasoduodenal tubes in a randomized controlled trial enrolling 151 patients undergoing esophagectomy. The overall complication rate was not significantly different, but the catheter-related complication rate was rather high; $35 \%$ of the patients in the jejunostomy group developed catheterrelated complications compared to $30 \%$ in the nasoduodenal tube group, which was not statistically significant. The authors concluded that both methods were effective, but they could not identify obvious advantages for any of the methods applied. Reviewing the present data conclusively, feeding jejunostomies do not seem to lead to increased complication rates, with the advantage of long-term feeding access and a possible reduction of pulmonary complications. Therefore jejunal insertion of a feeding tube during surgery should be recommended in order to provide enteral nutrition as early as possible.

\section{Immunonutrition}

The application of immunonutrition formulas has been increasingly investigated in upper gastrointestinal surgery. Immunonutrition is a feeding solution enriched with arginine, glutamine, omega-3 fatty acids and RNA. These solutions are considered to modulate immune responses and enhance antioxidant cell responses. Therefore, immunonutrition is supposed to reduce postoperative complications, which in turn is supposed to be beneficial for early 
recovery. A recent meta-analysis in gastric cancer patients revealed that immunonutrition may be beneficial for the induction of NK and CD4 cell responses as well as for the reduction of IL-6/TNF $\alpha$ levels and the increase of IgA and IgM. However, these effects did not translate into reduced complication rates or reduced length of hospital stay [51]. Another systematic review including esophageal surgeries found similar conclusions [52]. The reasons were related to the heterogeneity of the respective studies and small sample sizes. From an evidence-based point of view, immunonutrition does not substantially contribute to a significant reduction of postoperative complications in gastroesophageal surgery.

\section{Reconstruction Routes}

Reconstructing the gastrointestinal passage is the prerequisite to provide enteral nutrition to any patient undergoing resection for esophageal or gastroesophageal malignancy. However, different reconstruction techniques do not apply for patients undergoing transthoracic esophagectomy because usually the stomach is used as a substitute for the resected esophagus. In contrast, patients undergoing transhiatally extended gastrectomy for gastroesophageal junction cancers of Siewert type II and III are eligible for various reconstruction techniques. Several possibilities have been evaluated in the recent past. The most common techniques are the pouch reconstruction and the Roux-en-Y reconstructions. A meta-analysis by Gertler et al. [53] on thirteen randomized controlled trials demonstrated that pouch reconstructions provided several advantages, such as reduced dumping symptoms and reduced heartburn, while at the same time postoperative morbidity and mortality as much as postoperative length of hospital stay and operating times were not increased compared to a simple Roux-en-Y reconstruction. Most importantly, patients undergoing pouch reconstructions had improved eating capacity, increased body weight faster and revealed higher quality of life scores. Another meta-analysis on pouch sizes from China further concluded that small pouches demonstrated advantages over large pouches with regard to eating capacity per meal, while at the same time heartburn, dysphagia, vomiting and post-gastrectomy syndromes could be improved [54]. Conclusively, patients undergoing extended gastric resections should be considered for pouch reconstructions in order to control for weight loss and malnutrition.

\section{Postoperative Pancreatic Insufficiency}

Exocrine insufficiency of the pancreas is reported to be associated with weight loss and malnutrition after gastrointestinal surgery. The disruption of the stimulating pathways after gastroesophageal surgery is supposed to result in reduced output of pancreatic enzymes which leads to steatorrhea, diarrhea and impaired absorption of fat-soluble vitamins [55]. Friess et al. [56] investigated pancreatic function after total gastrectomy in 15 patients by a secretin-cerulein test and found that pancreatic secretion of trypsin, chymotrypsin and amylase was significantly reduced 3 months after surgery. Not only exocrine but also endocrine function was depressed after gastrectomy: all patients demonstrated not only impaired glucose tolerance but also reduced gastrin and pancreatic polypeptide. An older study by Armbrecht et al. [57] investigated the influence of postoperative pancreatic enzyme supplementation. Here, 15 patients either received pancreatic enzyme substitution after total gastrectomy or placebo. The authors demonstrated that stool consistency became more solid and fecal fat excretion was reduced in patients with massive steatorrhea (defined as content of free and esterified fatty acids in the stool $>350 \mathrm{ml} / 72 \mathrm{~h}$ ). A 
follow-up study by Brägelmann et al. [58] randomized 52 patients with a fecal fat output of $>14 \mathrm{~g} /$ day after total gastrectomy for cancer to either receive pancreatic enzymes or placebo. Interestingly there was no improvement of specific symptoms, but patients felt more comfortable after enzyme supplementation. Further, the median kilojoule intake per kilogram body weight was significantly higher in the placebo group and no differences in bowel habits or fat malassimilation were reported. The most recent study by Huddy et al. [59] examined 63 patients after esophagectomy who received pancreatic enzyme replacement, with exocrine pancreatic insufficiency proven by marked reduction of fecal elastase-1 excretion in the stool $(<200 \mu \mathrm{g} / \mathrm{g})$ or with moderate decrease $(200-500 \mu \mathrm{g} / \mathrm{g})$ accompanied by severe symptoms. The authors found that $16 \%$ of the patients demonstrated severe exocrine pancreatic insufficiency and failed to regain their preoperative weights. It was shown that symptomatic improvements were achieved in $90 \%$ of the cases and $80 \%$ either increased their weight or were able to keep it at a stable level. Patients with moderate decrease of fecal elastase- 1 revealed symptomatic benefit in only $42 \%$ of the cases, and only $17 \%$ were able to gain weight. Conclusively these data suggest that patients after esophageal or gastric resections for cancer should be tested for pancreatic exocrine insufficiency and treated by enzyme substitution if this is confirmed. The value of prophylactic enzyme replacement in oligosymptomatic or unproven pancreatic insufficiency has to be carefully evaluated in the future.

\section{Procedures in Patients Undergoing Non-Curative Treatments}

Feeding challenges are most pronounced in patients undergoing non-curative treatments due to metastatic disease or technical irresectability. These patients usually undergo chemotherapy or chemoradiation for palliation. Surgical resection is not indicated as long as no life-threatening situations, such as bleeding or perforation, occur. However, these patients are challenging regarding nutritional support. Due to progressive dysphagia in the beginning of their treatments, this group of patients is exposed to a high risk of malnutrition. Therefore, feeding access and local treatments are considered to be of utmost importance in order to preserve quality of life. Further, it was shown that malnutrition affects the effectiveness of chemotherapeutic treatment not only due to changes in the protein metabolism, but also leads to increased toxicity and in consequence to inadequate tumor control [16]. Several techniques have been proposed in the past to gain local tumor control, which are self-expanding metal stents (SEMS), thermal laser therapy, photodynamic therapy (PDT) and argon plasma coagulation in addition to chemotherapy and chemoradiation. A recent meta-analysis on 53 randomized controlled trials by Dai et al. [60] investigated dysphagia improvements for the above-mentioned treatment modalities.

SEMS versus Plastic Tube Stents. Seven trials investigated the outcome after SEMS insertion compared to plastic tube stents [60] and concluded that SEMS insertion was superior with regard to overall dysphagia improvement, although the result was not statistically significant. Regarding persistent or recurrent dysphagia there was a statistically significant benefit in favor of SEMS ( $p=0.017)$. The procedure mortality was reported to be higher in the plastic stent group $(p=0.019)$, the adverse events rate was significantly lower in the SEMS group and the initial hospital stay was significantly shorter in the latter. Quality of life and overall survival were not improved by SEMS insertion. Conclusively the authors stated that SEMS insertion can be considered superior to plastic stent insertion.

Stent versus Laser. Two trials investigating SEMS versus laser ablation were included in the above-mentioned meta-analysis [60]. The authors found that neither of the two procedures was superior to the other regarding dysphagia improvement, recurrent dysphagia and 
procedure-related mortality. There were no differences in quality of life and overall mortality. However, SEMS insertion had a higher procedure-related success rate and less patients had to undergo re-interventions. Regarding the evidence presented here, there is no obvious advantage for either technique. When laser application was compared to plastic stent insertion no differences could be identified regarding dysphagia improvement, procedure-related mortality, adverse event rate and overall survival.

SEMS versus Brachytherapy. Two trials reported on the outcomes of SEMS insertion compared to brachytherapy [60]. It was shown that SEMS insertion provided faster dysphagia improvement, which diminished over time. Brachytherapy was reported to provide tumor control for a longer time period and higher quality of life scores. Further, there was a significantly lower rate of procedure-related complications both in esophageal and in gastroesophageal cancer patients.

Laser versus Laser with or without either Brachytherapy or External Beam Radiation. Six trials investigated on the outcomes of laser therapy versus laser therapy combined with radiation therapy [60]. Radiation was delivered either by external beam or by brachytherapy. The meta-analysis on these trials found that laser therapy which added radiation increased the dysphagia-free period and reduced recurrent dysphagia without significantly improving survival.

Laser versus PDT. This analysis included two randomized controlled trials comparing laser versus PDT [60]. The results revealed no significant differences in dysphagia improvement, but repeated endoscopic interventions were less often required after PDT. Further PDT improved quality of life and dietary performance. However, overall survival was not improved by either of the two techniques.

Several combinations of the above-mentioned techniques were investigated in randomized trials. However, due to the heterogeneity of these studies, clear conclusions cannot be drawn from the existing evidence. The authors of a meta-analysis further concluded that SEMS insertion can be a reliable and fast method on palliating dysphagia symptoms whereas brachytherapy may be more effective regarding long-term control [60]. From the authors' point of view plastic tube insertion, chemotherapy and chemoradiation alone cannot be recommended for palliation. Combination therapies will have to be investigated more extensively in the future, with a special focus on quality of life and dysphagia improvement.

\section{Stent Insertion in the Neoadjuvant Setting}

Stent insertion was investigated by Jones and Griffiths [61] for patients undergoing neoadjuvant treatment for advanced esophageal cancers. The systematic review demonstrated a rapid improvement of dysphagia in all of the included studies. However, no significant improvement of nutritional status, defined by body weight and albumin levels, was noted. There was a considerable heterogeneity between the studies. The rate of patients proceeding to surgical resection varied extensively from 0 to $56 \%$. This however was attributable to disease progression and not to a decline in nutritional resources. Further, there was a considerable amount of stent-associated complications like migration and perforation, especially when the patients responded well to neoadjuvant treatment. The authors concluded that stent insertion for patients undergoing neoadjuvant treatment in esophageal cancers cannot be supported despite rapid improvement of dysphagia because the procedure did not translate into improved nutritional status, while patients developed a considerable amount of complications at the same time. 


\section{Conclusions}

Based on the data and literature presented in this review, these recommendations are supposed to overcome feeding challenges in patients undergoing esophageal and gastroesophageal cancer surgery:

(1) Adequate evaluation of malnutrition risk. Adequate identification of patients by screening tools such as the NRS 2002 or the SGA is a prerequisite to avoid malnutrition and malnourishment.

(2) Prevention of weight loss during multimodal therapy. Although the evidence is not conclusive, preoperative weight loss should be avoided to reduce postoperative morbidity by either enteral or parenteral nutrition. Stent insertion during neoadjuvant therapy does not improve nutritional status significantly and should be avoided in order to reduce procedureassociated complications.

(3) Consideration of reconstruction routes for faster weight gain. Pouch reconstructions in (extended) gastric resections should be considered for their obvious advantages in increased eating capacity, weight gain and quality of life. For esophageal cancer patients undergoing transthoracic resection with gastric conduit reconstruction, feeding jejunostomy should be applied to ensure early enteral nutrition.

(4) Prevention of postoperative complications. Postoperative complications should be avoided to improve nutritional status without delays. ERAS programs appear to be helpful, but evidence-based recommendations do not exist. Pancreatic enzyme replacement should be considered in patients unable to gain weight and with clinical symptoms of pancreatic exocrine insufficiency after appropriate testing.

(5) Conservation of the oral passage in palliative, unresectable and palliative patients. Early stent insertion (SEMS) is recommended in palliative patients to reduce dysphagia. Brachytherapy provides better long-term control and higher quality of life scores. There is no high-level evidence for multimodal combination therapies yet.

The data presented in this review provide some evidence that control for malnutrition may help improve not only quality of life but also patients' oncologic outcome. However, evidence-based data on various interventions can be considered scarce and modern clinical research should be encouraged by initiation of randomized controlled trials in order to reduce uncertainties in nutrition management of esophagogastric cancer patients.

\section{Disclosure Statement}

None of the authors has any conflicts of interest. No funds or grants or company gifts were received, nor has this article been written by a third party.

\section{References}

$>1$ Vandebroek AJ, Schrijvers D: Nutritional issues in anti-cancer treatment. Ann Oncol 2008;19(suppl 5):v52v55.

2 Bozzetti F: Nutritional support in patients with oesophageal cancer. Support Care Cancer 2010;18(suppl 2):S41-S50.

3 Fitzmaurice C, Dicker D, Pain A, et al: The Global Burden of Cancer 2013. JAMA Oncol 2015;1:505-527.

4 Pennathur A, Gibson MK, Jobe BA, et al: Oesophageal carcinoma. Lancet 2013;381:400-412.

5 Mariette C, Dahan L, Mornex F, et al: Surgery alone versus chemoradiotherapy followed by surgery for stage I and II esophageal cancer: final analysis of randomized controlled phase III trial FFCD 9901. J Clin Oncol 2014; 32:2416-2422.

6 Blazeby JM, Strong S, Donovan JL, et al: Feasibility RCT of definitive chemoradiotherapy or chemotherapy and surgery for oesophageal squamous cell cancer. Br J Cancer 2014;111:234-240. 
$\checkmark 7$ Conroy T, Galais MP, Raoul JL, et al: Definitive chemoradiotherapy with FOLFOX versus fluorouracil and cisplatin in patients with oesophageal cancer (PRODIGE5/ACCORD17): final results of a randomised, phase 2/3 trial. Lancet Oncol 2014;15:305-314.

-8 Crosby T, Hurt CN, Falk S, et al: Chemoradiotherapy with or without cetuximab in patients with oesophageal cancer (SCOPE1): a multicentre, phase 2/3 randomised trial. Lancet Oncol 2013;14:627-637.

9 van Hagen P, Hulshof MC, van Lanschot JJ, et al: Preoperative chemoradiotherapy for esophageal or junctional cancer. N Engl J Med 2012;366:2074-2084.

10 Stahl M, Walz MK, Stuschke M, et al: Phase III comparison of preoperative chemotherapy compared with chemoradiotherapy in patients with locally advanced adenocarcinoma of the esophagogastric junction. J Clin Oncol 2009;27:851-856.

$>11$ Tepper J, Krasna MJ, Niedzwiecki D, et al: Phase III trial of trimodality therapy with cisplatin, fluorouracil, radiotherapy, and surgery compared with surgery alone for esophageal cancer: CALGB 9781. J Clin Oncol 2008;26:1086-1092.

12 Kelsen DP, Winter KA, Gunderson LL, et al: Long-term results of RTOG trial 8911 (USA Intergroup 113): a random assignment trial comparison of chemotherapy followed by surgery compared with surgery alone for esophageal cancer. J Clin Oncol 2007;25:3719-3725.

13 Bedenne L, Michel P, Bouche 0, et al: Chemoradiation followed by surgery compared with chemoradiation alone in squamous cancer of the esophagus: FFCD 9102. J Clin Oncol 2007;25:1160-1168.

14 Burmeister BH, Smithers BM, Gebski V, et al: Surgery alone versus chemoradiotherapy followed by surgery for resectable cancer of the oesophagus: a randomised controlled phase III trial. Lancet Oncol 2005;6:659668.

15 Bower MR, Martin RC 2nd: Nutritional management during neoadjuvant therapy for esophageal cancer. J Surg Oncol 2009;100:82-87.

16 Murry DJ, Riva L, Poplack DG: Impact of nutrition on pharmacokinetics of anti-neoplastic agents. Int J Cancer Suppl 1998;11:48-51.

17 Gaur P, Blackmon SH: Jejunal graft conduits after esophagectomy. J Thorac Dis 2014;6(suppl 3):S333-S340.

18 Bailey SH, Bull DA, Harpole DH, et al: Outcomes after esophagectomy: a ten-year prospective cohort. Ann Thorac Surg 2003;75:217-222; discussion 222.

19 Noble F, Curtis N, Harris S, et al: Risk assessment using a novel score to predict anastomotic leak and major complications after oesophageal resection. J Gastrointest Surg 2012;16:1083-1095.

20 Bozzetti F: Screening the nutritional status in oncology: a preliminary report on 1,000 outpatients. Support Care Cancer 2009;17:279-284.

21 Daly JM, Fry WA, Little AG, et al: Esophageal cancer: results of an American College of Surgeons Patient Care Evaluation Study. J Am Coll Surg 2000;190:562-572; discussion 572-573.

22 Martin L, Lagergren P: Long-term weight change after oesophageal cancer surgery. Br J Surg 2009;96:13081314.

23 Martin L, Jia C, Rouvelas I, et al: Risk factors for malnutrition after oesophageal and cardia cancer surgery. Br J Surg 2008;95:1362-1368.

24 Correia MI, Waitzberg DL: The impact of malnutrition on morbidity, mortality, length of hospital stay and costs evaluated through a multivariate model analysis. Clin Nutr 2003;22:235-239.

-25 Schiesser M, Muller S, Kirchhoff P, et al: Assessment of a novel screening score for nutritional risk in predicting complications in gastro-intestinal surgery. Clin Nutr 2008;27:565-570.

26 Baldwin C, Spiro A, Ahern R, et al: Oral nutritional interventions in malnourished patients with cancer: a systematic review and meta-analysis. J Natl Cancer Inst 2012;104:371-385.

-27 van der Schaaf MK, Tilanus HW, van Lanschot JJ, et al: The influence of preoperative weight loss on the postoperative course after esophageal cancer resection. J Thorac Cardiovasc Surg 2014;147:490-495.

-28 Zhang SS, Yang H, Luo KJ, et al: The impact of body mass index on complication and survival in resected oesophageal cancer: a clinical-based cohort and meta-analysis. Br J Cancer 2013;109:2894-2903.

29 Skipworth J, Foster J, Raptis D, et al: The effect of preoperative weight loss and body mass index on postoperative outcome in patients with esophagogastric carcinoma. Dis Esophagus 2009;22:559-563.

-30 Ikeda M, Natsugoe S, Ueno S, et al: Significant host- and tumor-related factors for predicting prognosis in patients with esophageal carcinoma. Ann Surg 2003;238:197-202.

-31 Filip B, Scarpa M, Cavallin F, et al: Postoperative outcome after oesophagectomy for cancer: nutritional status is the missing ring in the current prognostic scores. Eur J Surg Oncol 2015;41:787-794.

-32 Goh SL, De Silva RP, Dhital K, et al: Is low serum albumin associated with postoperative complications in patients undergoing oesophagectomy for oesophageal malignancies? Interact Cardiovasc Thorac Surg 2015; 20:107-113.

-33 Skipper A, Ferguson M, Thompson K, et al: Nutrition screening tools: an analysis of the evidence. JPEN J Parenter Enteral Nutr 2012;36:292-298.

34 Guo W, Ou G, Li X, et al: Screening of the nutritional risk of patients with gastric carcinoma before operation by NRS 2002 and its relationship with postoperative results. J Gastroenterol Hepatol 2010;25:800-803.

-35 Ryu SW, Kim IH: Comparison of different nutritional assessments in detecting malnutrition among gastric cancer patients. World J Gastroenterol 2010;16:3310-3317.

-36 Gavazzi C, Colatruglio S, Sironi A, et al: Importance of early nutritional screening in patients with gastric cancer. Br J Nutr 2011;106:1773-1778. 
Omidvari AH, Vali Y, Murray SM, et al: Nutritional screening for improving professional practice for patient outcomes in hospital and primary care settings. Cochrane Database Syst Rev 2013;6:CD005539.

38 van Bokhorst-de van der Schueren MA, Guaitoli PR, Jansma EP, et al: Nutrition screening tools: does one size fit all? A systematic review of screening tools for the hospital setting. Clin Nutr 2014;33:39-58.

39 Braga M, Ljungqvist O, Soeters P, et al: ESPEN Guidelines on Parenteral Nutrition: surgery. Clin Nutr 2009;28: 378-386.

40 Peter JV, Moran JL, Phillips-Hughes J: A metaanalysis of treatment outcomes of early enteral versus early parenteral nutrition in hospitalized patients. Crit Care Med 2005;33:213-220; discussion 260-261.

41 Harvey SE, Parrott F, Harrison DA, et al: Trial of the route of early nutritional support in critically ill adults. N Engl J Med 2014;371:1673-1684.

-42 Doig GS, Simpson F, Sweetman EA, et al: Early parenteral nutrition in critically ill patients with short-term relative contraindications to early enteral nutrition: a randomized controlled trial. JAMA 2013;309:21302138.

43 Peng J, Cai J, Niu ZX, et al: Early enteral nutrition compared with parenteral nutrition for esophageal cancer patients after esophagectomy: a meta-analysis. Dis Esophagus 2015, Epub ahead of print.

44 Hirai T, Yamashita Y, Mukaida H, et al: Poor prognosis in esophageal cancer patients with postoperative complications. Surg Today 1998;28:576-579.

45 Ott K, Bader FG, Lordick F, et al: Surgical factors influence the outcome after Ivor-Lewis esophagectomy with intrathoracic anastomosis for adenocarcinoma of the esophagogastric junction: a consecutive series of 240 patients at an experienced center. Ann Surg Oncol 2009;16:1017-1025.

-46 Carrott PW, Markar SR, Kuppusamy MK, et al: Accordion Severity Grading System: assessment of relationship between costs, length of hospital stay, and survival in patients with complications after esophagectomy for cancer. J Am Coll Surg 2012;215:331-336.

47 Findlay JM, Gillies RS, Millo J, et al: Enhanced recovery for esophagectomy: a systematic review and evidencebased guidelines. Ann Surg 2014;259:413-431.

$\checkmark 48$ Torres Junior LG, de Vasconcellos Santos FA, Correia MI: Randomized clinical trial: nasoenteric tube or jejunostomy as a route for nutrition after major upper gastrointestinal operations. World J Surg 2014;38:22412246.

49 Sica GS, Sujendran V, Wheeler J, et al: Needle catheter jejunostomy at esophagectomy for cancer. J Surg Oncol 2005;91:276-279.

-50 Han-Geurts IJ, Hop WC, Verhoef C, et al: Randomized clinical trial comparing feeding jejunostomy with nasoduodenal tube placement in patients undergoing oesophagectomy. Br J Surg 2007;94:31-35.

51 Song GM, Tian X, Liang H, et al: Role of enteral immunonutrition in patients undergoing surgery for gastric cancer: a systematic review and meta-analysis of randomized controlled trials. Medicine (Baltimore) 2015; 94:e1311.

52 Mabvuure NT, Roman A, Khan OA: Enteral immunonutrition versus standard enteral nutrition for patients undergoing oesophagogastric resection for cancer. Int J Surg 2013;11:122-127.

53 Gertler R, Rosenberg R, Feith M, et al: Pouch versus no pouch following total gastrectomy: meta-analysis and systematic review. Am J Gastroenterol 2009;104:2838-2851.

-54 Dong HL, Huang YB, Ding XW, et al: Pouch size influences clinical outcome of pouch construction after total gastrectomy: a meta-analysis. World J Gastroenterol 2014;20:10166-10173.

55 Friess H, Michalski CW: Diagnosing exocrine pancreatic insufficiency after surgery: when and which patients to treat. HPB (Oxford) 2009;11(suppl 3):7-10.

56 Friess H, Bohm J, Muller MW, et al: Maldigestion after total gastrectomy is associated with pancreatic insufficiency. Am J Gastroenterol 1996;91:341-347.

57 Armbrecht U, Lundell L, Stockbrugger RW: The benefit of pancreatic enzyme substitution after total gastrectomy. Aliment Pharmacol Ther 1988;2:493-500.

58 Brägelmann R, Armbrecht U, Rosemeyer D, et al: The effect of pancreatic enzyme supplementation in patients with steatorrhoea after total gastrectomy. Eur J Gastroenterol Hepatol 1999;11:231-237.

59 Huddy JR, Macharg FM, Lawn AM, et al: Exocrine pancreatic insufficiency following esophagectomy. Dis Esophagus 2013;26:594-597.

60 Dai Y, Li C, Xie Y, et al: Interventions for dysphagia in oesophageal cancer. Cochrane Database Syst Rev 2014; 10:CD005048.

61 Jones CM, Griffiths EA: Should oesophageal stents be used before neo-adjuvant therapy to treat dysphagia in patients awaiting oesophagectomy? Best evidence topic (BET). Int J Surg 2014;12:1172-1180. 